\title{
INVENTÁRIO DOS EQUINODERMOS DO ESTADO DE SANTA CATARINA, BRASIL
}

\author{
Xavier, L. A. R. \\ Universidade do Vale do Itajaí, UNIVALI. \\ larx@ibest.com.br
}

\begin{abstract}
Xavier, L. A. R. 2010. Checklist of Echinodermata in Santa Catarina State, Brazil. Braz. J. Aquat. Sci. Technol. 14(2): 73-78. ISSN 1808-7035. The phylum Echinodermata includes approximately 7000 living species divided into five classes. In Brazil, 335 species are recorded, but there is much to be studied on this phylum. This study aimed to identify the occurrence of species of Echinodermata of the State of Santa Catarina (Brazil), which are represented by a total of 34 species. Of these, 09 belong to the class Asteroidea, 14 to Ophiuroidea, 08 to Echinoidea, 02 to Holoturoidea and 01 to Crinoidea. 12 species listed in this article are listed as threatened of extinction.
\end{abstract}

Keywords: Biodiversity; Richness; Brazil.

\section{INTRODUÇÃO}

Os equinodermos encontram-se entre os invertebrados marinhos mais conhecidos e seus representantes tornaram-se símbolos desse ambiente (Ruppert \& Barnes, 1996). São animais predominantemente marinhos (alguns toleram água salobra), podendo ser encontrados em todos os oceanos, latitudes e profundidades, da zona entremarés às regiões abissais, sendo mais abundantes na região tropical do que nas águas polares (Magalhães et al., 2005). Chegam a compor $95 \%$ da biomassa total nas regiões abissais (Brusca \& Brusca, 2003).

São importantes componentes do bentos costeiro ocupando diversos tipos de substratos (rochosos, arenosos, lodosos, em madeira submersa ou em epibiose), se adaptando para fixar-se a eles (Magalhães et al., 2005).

Constituem cerca de 7000 espécies vivas conhecidas, distribuídas em 5 classes (Brusca \& Brusca, 2003) e 13000 espécies fósseis (Tommasi, 1999). No Brasil eram registradas 329 espécies (Tommasi, 1999), mas esse número vem crescendo com pelo menos seis novos registros de ocorrência de Ophiuroidea por Borges et al. (2002) e Borges \& Amaral (2007), elevando então esse número para 335 espécies no Brasil.

Apesar dos esforços de alguns pesquisadores, a biologia e ecologia desses animais ainda são pouco conhecidas no Brasil, sendo que a maioria dos trabaIhos ocorre sob um enfoque de avaliação geral, como simples parte integrante da fauna bêntica de uma determinada região. Análises mais aprofundadas desse grupo são importantes, sobre diversos aspectos, como por exemplo, equinodermos carnívoros (Asteroidea) que são predadores ativos em cultivos de moluscos, e herbívoros (Echinoidea) que são consumidores ativos de marismas e bancos de algas.
Rodrigues et al. (2004) mencionam que as comunidades bióticas costeiras, incluindo os equinodermos, estão sujeitas não só aos estresses naturais como também aos distúrbios de origem antrópica, causados por diferentes fontes de poluição. Esses animais são um grupo sensível às características ambientais locais e, conseqüentemente um dos primeiros a serem prejudicados pelo déficit de oxigênio na água (Diaz, R. J. \& Rosemberg, R., 1995 apud Tommasi, 1999). Assim, podem representar excelentes indicadores ambientais.

Outra questão importante é a utilização crescente desses animais na gastronomia, em especial as gônadas de ouriços-do-mar e pele de holotúrias (o trepang) (Tommasi, 1999). Também, por serem animais vistosos e coloridos, são alvo constante da utilização e exploração para zooartesanatos, ou artesanatos produzidos com animais ou parte deste, para fins ornamentais (Magalhães et al., 2005),

Com base nessas lacunas de conhecimento, o presente artigo teve por objetivo listar as espécies que ocorrem no estado de Santa Catarina, a partir de revisões e levantamentos bibliográficos em periódicos especializados.

\section{MATERIAIS E MÉTODOS}

Foi feito um levantamento bibliográfico, em resumos de congressos brasileiros de zoologia e congressos brasileiros de oceanografia, e em diversos bancos de dados disponíveis na internet, selecionando resumos, artigos, monografias, dissertações e teses. Também foi consultada a lista de espécies ameaçadas de extinção publicada pelo Ministério do Meio Ambiente. 


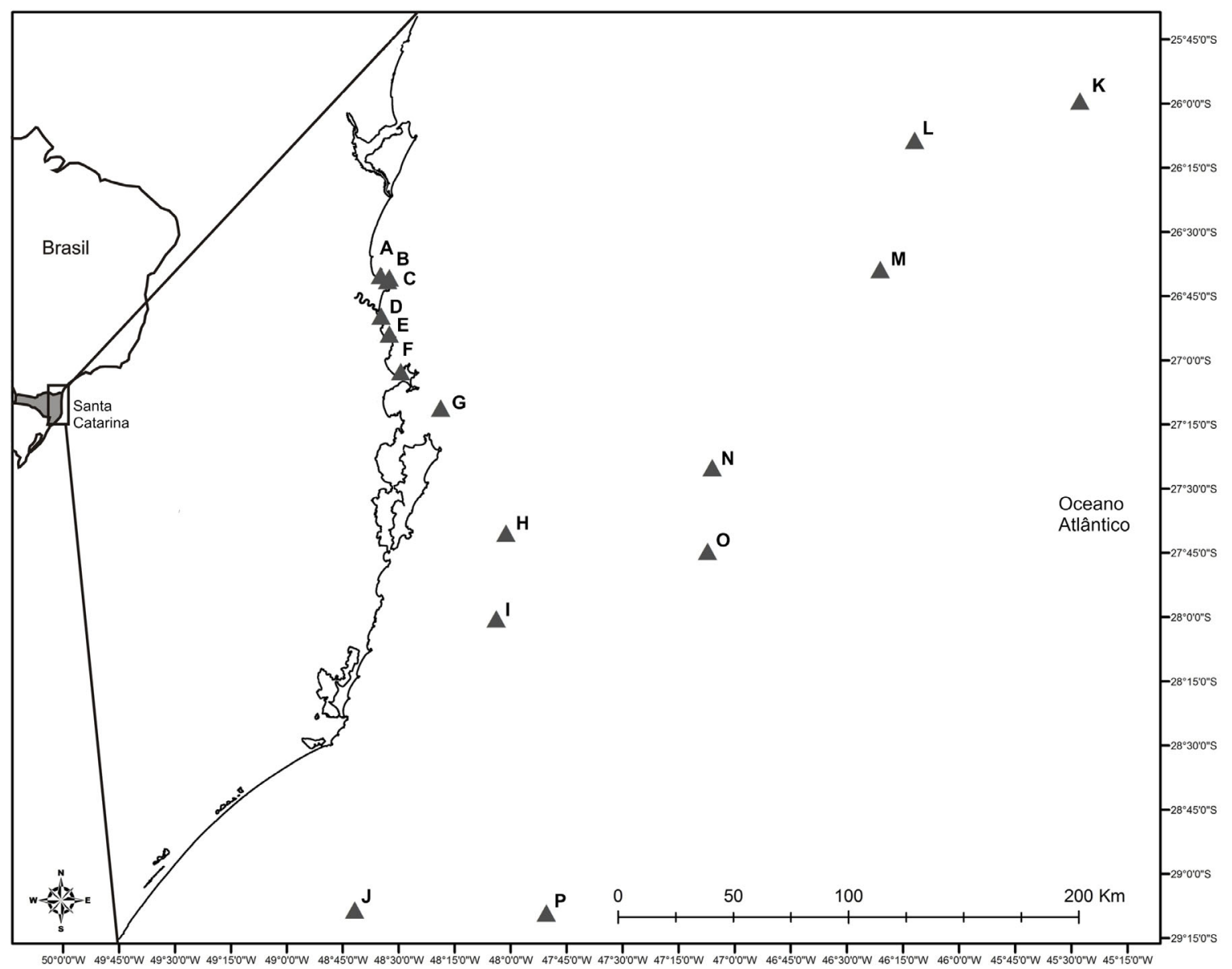

Figura 1 - Mapa de Santa Catarina com os locais onde equinodermos foram coletados segundo os trabalhos citados na Tabela 1. A - Praia do Quilombo - Penha (SC); B - Armação do Itapocoroy - Penha (SC); C - Praia da Paciência - Penha (SC); D - Praia de Cabeçudas - Itajaí (SC); E - Praia das Laranjeiras - Balneário Camboriu (SC); F - Ilha de João da Cunha - Porto Belo (SC); G - Ilha do Arvoredo (SC); H Estação 6789; I - Estação 6794; J - Estação 6814; K - Estação 6705; L - Estação 6698; M - Estação 6693; N - Estação 6786; O - Estação 6791; P - Estação 6811.

Após o levantamento dessas publicações, foram triados e selecionados trabalhos que mencionassem a ocorrência de espécies no estado de Santa Catarina.

\section{RESULTADOS E DISCUSSÃO}

Santa Catarina tem $450 \mathrm{~km}$ de costa oceânica e muitas lacunas de conhecimento sobre a fauna dos Equinodermos do estado.

A revisão bibliográfica para este trabalho resultou numa lista taxonômica de equinodermos referenciados para o estado de Santa Catarina, apresentados na Tabela 1. Foram citadas 34 espécies, distribuídas em 21 famílias, representando as cinco classes.

Destas 34 espécies, 23 foram registradas em Santa Catarina (Tabela 2), e os locais de suas coletas são apresentados na Figura 1. As outras 11 espécies são apenas sugeridas para o Estado, estando Santa
Catarina dentro da distribuição geográfica descrita para cada espécie e/ou por estarem relacionadas na lista brasileira de espécies ameaçadas de extinção (MMA, 2004), mas nenhum trabalho descreveu ou citou um local específico onde essas tenham sido encontradas. Os Ophiuroidea foram a classe com maior número de espécies referenciadas (14 espécies), seguidos dos Asteroidea (9 espécies) e dos Echinoidea (8 espécies). Os Holoturoidea tiveram 2 espécies referenciadas para o estado e a Classe Crinoidea apenas 1 espécie.

Da classe Ophiuroidea destaca-se a espécie Amphiura deichmanae Tommasi, 1965, que tem a localidade tipo descrita para a espécie na praia de Cabeçudas (Itajai-SC) (Tommasi, 1965).

As espécies Amphilimna mirabilis (H.L.Clark, 1941), Amphiura complanata Ljungman, 1867, Amphiura flexuosa Ljungman, 1867, Amphiura mülleri MarktannerTurneretsche, 1887, Ophiacantha cosmica Lyman 1878, Ophiostriatus striatus (Mortensen, 1933), Ophiomastus 
Tabela 1- Lista taxonômica das espécies referenciadas e/ou sugeridas para Santa Catarina. a - Amaral et al., 2008; b - Bahr, 2000; c - Borges \& Amaral, 2007 ; d - Borges et al., 2002; e - Borzone, 1993; f - Couto, 1998; g - Giordano, 1986; h - Lunardon-Branco et al., 2004; i - Marchiori et al., 1996; j - Mendes et al., 2004; k - MMA, 2004; I - Monteiro, 1998; m - Netto, 2001; n - Santana \& Silva, 1996; o - Tommasi, 1965; p - Tommasi, 1999; q - Vidolin et al., 2002; $r$ - Xavier, 2005. * - Espécies citadas para o estado, mas sem comprovação de registro.

\begin{tabular}{|c|c|c|c|}
\hline CLASSE & FAMÍLIA & ESPÉCIE & REFERÊNCIA \\
\hline \multirow[t]{9}{*}{ Asteroidea } & Asteroidae & Asterina stellifera (Möbius, 1859) & akpr \\
\hline & Astropectinidae & Astropecten brasiliensis Müller \& Troschel, 1842 & akpr* \\
\hline & & Astropecten marginatus Gray, 1840 & $a^{*}$ \\
\hline & & Astropecten cingulatus Sladen, 1889 & $a^{*}$ \\
\hline & Echinasteridae & Echinaster (Othilia) brasiliensis Müller \& Troschel, 1842 & a ikpr \\
\hline & Luidiidae & Luidia Iudwigi Bell, 1917 & $a^{*}$ \\
\hline & & Luidia clathrata (Say, 1825) & $\mathrm{ah}$ \\
\hline & & Luidia senegalensis (Lamarck, 1816) & $\mathrm{ah}$ \\
\hline & Oreasteridae & Oreaster reticulatus (Linnaeus, 1758) & $a k^{*}$ \\
\hline Crinoidea & Tropiometridae & Tropiometra carinata (Lamark, 1816) A. H. Clark, 1907 & $m^{*}$ \\
\hline \multirow[t]{8}{*}{ Echinoidea } & Arbaciidae & Arbacia lixula (Linnaeus, 1758) & gir \\
\hline & Cidaridae & Eucidaris tribuloides (Lamarck, 1816) & a i \\
\hline & Clypeasteridae & Astropyga nuptialis (A. H. Clark, 1934) & $p^{*}$ \\
\hline & & Clypeaster subdepressus (Gray, 1825) & $\mathrm{i}$ \\
\hline & Echinidae & Paracentrotus gaimardi (Blainville, 1825) & a ik \\
\hline & Echinometridae & Echinometra lucunter (Linnaeus, 1758) & b in $r$ \\
\hline & Mellitidae & Mellita quinquiesperforata (Leske, 1778) & $e^{*}$ \\
\hline & Toxopneustidae & Lytechinus variegatus (Lamarck, 1816) & fir \\
\hline \multirow[t]{2}{*}{ Holoturoidea } & Holothuridae & Holothuria grisea Selenka, 1867 & $\mathrm{jmp}$ \\
\hline & Stichopodidae & Isostichopus badionotus Selenka, 1867 & a k m* \\
\hline \multirow[t]{14}{*}{ Ophiuroidea } & Amphiuridae & Amphilimna mirabilis (H.L.Clark, 1941) & $d$ \\
\hline & & Amphilimna olivacea (Lyman, 1869) & $d^{*}$ \\
\hline & & Amphioplus albidus (Ljungman, 1867) & $d^{*}$ \\
\hline & & Amphiura complan ata Ljungman, 1867 & $d$ \\
\hline & & Amphiura deichmanae Tommasi, 1965 & o \\
\hline & & Amphiura flexuosa Ljungman, 1867 & $d$ \\
\hline & & Amphiura mülleri Marktanner -Turneretsche, 1887 & $d$ \\
\hline & Ophiacanthidae & Ophiacantha cosmica Lyman 1878 & $d$ \\
\hline & Ophiolepididae & Ophiomusium eburneum Lyman, 1869 & c \\
\hline & Ophioleucidae & Ophiostriatus striatus (Mortensen, 1933) & $d$ \\
\hline & Ophiothricidae & Ophiothrix angulata (Say, 1825) & I $r$ \\
\hline & Ophiuroidae & Ophiomastus satelitae Tommasi \& Abreu, 1974 & d \\
\hline & & Ophiura (Ophiuroglypha) clemens (Koehler, 1904) & c \\
\hline & & Ophiura ljungmani (Lyman, 1878) & d \\
\hline
\end{tabular}

satelitae Tommasi \& Abreu, 1974 e Ophiura ljungmani (Lyman, 1878) foram coletadas e identificadas por Borges et al., (2002) em estações que cobriam a plataforma externa e parte do talude, entre as isóbatas de 60 e $810 m$ de profundidade, incluindo Santa Catarina.

Borges \& Amaral (2007) registraram a ocorrência das espécies Ophiura (Ophiuroglypha) clemens (Koehler, 1904) e Ophiomusium eburneum Lyman, 1869, sendo inclusive o primeiro registro das espécies para o Brasil.

A espécie Ophiothrix angulata (Say, 1825) (Família Ophiothricidae), é citada para Santa Catarina por Monteiro (1998), que menciona a espécie como sendo uma das mais comuns de ofiúros da costa brasileira. Esta espécie também foi encontrada por Xavier (2005), na região de entremarés da praia da Paciência, Penha - SC.
Das espécies de Echinoidea citadas para Santa Catarina, Echinometra lucunter (Linnaeus, 1758) é considerada a mais abundante do litoral brasileiro, e a de maior aceitação para fins alimentícios. Por isso muitos trabalhos científicos tem sido realizados a seu respeito, no que diz respeito à ecologia, parâmetros ambientais, nutrição, cultivo, fisiologia, etc. A espécie é referenciada para Santa Catarina por Santana \& Silva, (1996) e registrada por Marchiori et al. (1996), Bahr (2000) e Xavier (2005).

Xavier (2005) também registrou a ocorrência dos equinóides Arbacia lixula (Linnaeus, 1758) e Lytechinus variegatus (Lamarck, 1816). L. variegatus foi registrada também por Marchiori et al. (1996) na llha do Arvoredo (SC) e referenciada para SC por Couto (1998), sendo considerada comum a todo o litoral brasileiro.

O Asteroidea Echinaster (Othilia) brasiliensis Müller \& Troschel, 1842 tem sua presença indicada no 
Tabela 2 - Lista das espécies com suas respectivas ocorrências. As letras representam os locais (ver Figura 1).

\begin{tabular}{lll}
\hline \hline CLASSE & ESPÉCIE & OCORRÊNCIA \\
\hline Asteroidea & Asterina stellifera (Möbius, 1859) & A C \\
& Echinaster (Othilia) brasiliensis Müller \& Troschel, 1842 & C G \\
& Luidia clathrata (Say, 1825) & B \\
& Luidia senegalensis (Lamarck, 1816) & $\mathrm{B}$ \\
Echinoidea & Arbacia lixula (Linnaeus, 1758) & $\mathrm{C}$ \\
& Eucidaris tribuloides (Lamarck, 1816) & $\mathrm{G}$ \\
& Clypeaster subdepressus (Gray, 1825) & $\mathrm{G}$ \\
& Paracentrotus gaimardi (Blainville, 1825) & $\mathrm{G}$ \\
& Echinometra lucunter (Linnaeus, 1758) & $\mathrm{C} \mathrm{E} \mathrm{F} \mathrm{G}$ \\
& Lytechinus variegatus (Lamarck, 1816) & $\mathrm{C} \mathrm{G}$ \\
Holoturoidea & Holothuria grisea Selenka, 1867 & $\mathrm{A} \mathrm{B}$ \\
& Amphilimna mirabilis (H.L.Clark, 1941) & $\mathrm{L}$ \\
& Amphiura complanata Ljungman, 1867 & $\mathrm{H} \mathrm{M}$ \\
& Amphiura deichmanae Tommasi, 1965 & $\mathrm{D}$ \\
& Amphiura flexuosa Ljungman, 1867 & $\mathrm{H} \mathrm{I} \mathrm{J} \mathrm{M}$ \\
& Amphiura mülleri Marktanner -Turneretsche, 1887 & $\mathrm{O}$ \\
& Ophiacantha cosmica Lyman 1878 & $\mathrm{M} \mathrm{N} \mathrm{P}$ \\
& Ophiomusium eburneum Lyman, 1869 & $\mathrm{K} \mathrm{M} \mathrm{N}$ \\
& Ophiostriatus striatus (Mortensen, 1933) & $\mathrm{M}$ \\
& Ophiothrix angulata (Say, 1825) & $\mathrm{C}$ \\
& Ophiomastus satelitae Tommasi \& Abreu, 1974 & $\mathrm{MN}$ \\
& Ophiura (Ophiuroglypha) clemens (Koehler, 1904) & $\mathrm{MN}$ \\
& Ophiura ljungmani (Lyman, 1878) & $\mathrm{M}$ \\
\hline \hline
\end{tabular}

litoral catarinense por Tommasi (1999) e foi registrada por Marchiori et al. (1996) e Xavier (2005). Asterina stellifera (Möbius, 1859) é referenciada para o estado por Vidolin et al. (2002) e Xavier (2005) em 2 áreas distintas do Município de Penha (SC).

Do mesmo Município, vem o registro dos asteróides Luidia clathrata (Say, 1825) e Luidia senegalensis (Lamarck, 1816) por Lunardon-Branco et al. (2004), os quais encontraram esses espécimes em redes de arrasto na Armação do Itapocoroy.

O Holoturoidea Holothuria grisea Selenka, 1867 foi referenciado para o Estado por Netto (2001) e registrado por Mendes et al. (2004) e Vidolin et al. (2002), ambos os registros no município de Penha (SC). Esta espécie é citada como sendo a holotúria mais abundante no Brasil.

Os asteroidea Astropecten brasiliensis Müller \& Troschel, 1842, Astropecten marginatus Gray, 1840, Astropecten cingulatus Sladen, 1889, Luidia ludwigi Bell, 1917 e Oreaster reticulatus (Linnaeus, 1758), o Crinoidea Tropiometra carinata (Lamark, 1816) A. H. Clark, 1907, os Echinoidea Astropyga nuptialis (A. H. Clark, 1934) e Mellita quinquiesperforata (Leske, 1778), o holoturoidea Isostichopus badionotus Selenka, 1867 e os Ophiuroidea Amphilimna olivacea (Lyman, 1869), Amphioplus albidus (Ljungman, 1867) foram citados por Amaral et al. (2008), Borges et al. (2002), Borzone (1993), MMA (2004), Netto (2001) e Tommasi (1999), sugerindo suas ocorrências em Santa Catarina, mas 76 nenhuma comprovação de registro para o estado foi encontrado (Tabela 1).

Essas lacunas de informações sobre o filo podem estar relacionadas com a falta de especialistas na região e evidencia a necessidade de mais trabalhos relacionados com o grupo no estado. Prova disso, é o registro de seis novas ocorrências de espécies de ofiuróides no Brasil (Borges et al., 2002; Borges \& Amaral, 2007), das quais 4 foram registradas para Santa Catarina.

Migotto \& Marques (2003) avaliando o estado de conhecimento da diversidade biológica no Brasil demonstraram em seus resultados que existe muito a ser revisto e visto sobre este grupo no Brasil, principalmente por se tratar de um dos mais importantes na comunidade bêntica.

\section{REFERÊNCIAS}

Amaral, A. C. Z.; Riberio, C. V.; Mansur, M. C. D.; Santos, S. B. dos; Avelar, W. E. P.; Cascon, H. M.; Leite, F. P. P.; Melo, G. A. S.; Coelho, P. A.; Buckup, G. B.; Buckup, L.; Ventura, C. R. R. \& Tiago, C. G. 2008. Invertebrados Aquáticos. In: MMA, Livro Vermelho da Fauna Brasileira ameaçada de extinção. Volume II. 
Bahr, J. 2000. Dinâmica de ouriços-do-mar em quatro localidades do litoral norte de Santa Catarina: variação temporal na abundância, crescimento e reprodução. Monografia de Graduação em Ciências Biológicas. Centro de Ciências Exatas e Naturais. FURB. Blumenau (SC). $36 \mathrm{p}$.

Borges, M. \& Amaral, A. C. Z. 2007. Ophiuroidea (Echinodermata): quatro novas ocorrências para o Brasil. Revista Brasileira de Zoologia 24 (4): 855864.

Borges, M.; Monteiro, A. M. G. \& Amaral, A. C. Z. 2002. Taxonomy of Ophiuroidea (Echinodermata) from the continental shelf and slope of the southern and southeastern Brazilian coast. Biota Neotropica 2(2): 1-69.

Borzone, C. A. 1993. Spatial distribution and growth of Mellita quinquiesperforata (Leske, 1778) on a sandy beach of southern Brazil. Nerítica 7 (1/2): 87-100.

Brusca, R. C \& Brusca, G. J. 2003. Invertebrates. 2.ed. Sunderland: Sinauer Associates, xix, $936 \mathrm{p}$.

Couto, E. C. G. 1998. Hábito alimentar do Ouriço-doMar Lytechinus variegatus (Lamarck, 1816) (Echinodermata: Echinoidea) e distribuição de macroalgas no Saco do Limoeiro (llha do Mel-PR). Resumos do XXII Congresso Brasileiro de Zoologia. Universidade Federal Rural de Pernambuco, Recife (PE). P. 197.

Giordano, F. 1986. Ouriços do sublitoral rochoso da região de São Sebastião, SP: uma abordagem ecológica. Tese de Mestrado em Ecologia, Instituto de Biologia. Universidade Estadual de Campinas, Campinas (SP). 130p.

Lunardon-Branco, M. J.; Branco, J. O. \& Verani, J. R.. 2004. Relações tróficas entre macroinvertebrados e peixes na Enseada da Armação do Itapocoroy, Penha, SC Resumos do Congresso Brasileiro de Oceanografia 2004 - XVI Semana Nacional de Oceanografia - UNIVALI - Itajaí - SC. P. 74.

Magalhães, W. F.; Martins, L. R. \& Alves, O. F. de S. 2005. Inventário dos do Estado da Bahia. Braz. J. Aquat. Sci. Technol., 9 (1): 61-65.

Marchiori, M. O.; Kohlmann, C. \& Fontoura, N. F. 1996. Densidade populacional e distribuição de Echinoidea e Asteroidea da llha do Arvoredo, Santa Catarina, Brasil. Resumos do XXI Congresso Brasileiro de Zoologia, Universidade Federal do Rio Grande do Sul, Porto Alegre (RS). 137pp.

Marenzi, A. W. C. \& Ribeiro, F. F. 2004. Subsídios para o desenvolvimento de uma tecnologia de cultivo do ouriço-do-mar, Echinometra lucunter (LINNAEUS, 1758). Resumos do Congresso Brasileiro de Oceanografia-XVI Semana Nacional de Oceanografia - UNIVALI - Itajaí - SC. P. 143.

Mendes, F. M.; Marenzi, A. W. C. \& Di Domenico, M. 2004. Caracterização de aspectos populacionais de
Holothuria grisea (Echinodermata, Holothuroidea) na Enseada da Armação do Itapocoroy, Penha - SC, Brasil. Resumos do Congresso Brasileiro de Oceanografia-XVI Semana Nacional de OceanografiaUNIVALI - Itajaí - SC. P. 97.

Migotto, A.E. \& Marques, A.C. 2003. Avaliação do estado de conhecimento da diversidade biológica do Brasil. Invertebrados Marinhos, versão preliminar. Ministério do Meio Ambiente. 87p.

MMA - Ministério Do Meio Ambiente. 2004. Lista das Espécies de Fauna Ameaçadas de Extinção. http:/ /www.mma.gov.br/estruturas/179/_arquivos/ in_mma_005_04_179.pdf (Acesso em 01 de julho de 2009).

Monteiro, A. M. G. 1998. Avaliação das espécies de ofiuróides (Echinodermata) conhecidas nas costa brasileira. Resumos do XXII Congresso Brasileiro de Zoologia. . Universidade Federal Rural de Pernambuco, P. 197

Netto, L. F. 2001. Echinodermata da zona entre marés do Canal de São Sebastião, SP. Monografia de Bacharelado em Ciências Biológicas - USP, Ribeirão Preto-SP. 70p.

Rodrigues, L. F. T.; Verde, W. G. V.; Silva, C. C. da \& Castro, G. A. de. 2004. Macrofauna bentônica do mesolitoral de costões rochosos da Baía de Beneventes, Espírito Santo. II Costa Azul e dos Namorados (Iriri). Resumos do Congresso Brasileiro de Oceanografia-XVI Semana Nacional de Oceanografia - UNIVALI - Itajaí - SC. P. 35.

Ruppert, E. \&. Barnes, R. D. 1996. Zoologia dos Invertebrados. 6ª . Ed. São Paulo: Editora Roca. 1028p.

Santana, I. C. H. \& Silva, J. R. F. da. 1996. Aspectos da fertilização e clivagens iniciais de Echinometra lucunter (LINNAEUS, 1758) (Echinodermata: Echinoidea: Echinometridae) em laboratório. Resumos do XXI Congresso Brasileiro de Zoologia. Universidade Federal do Rio Grande do Sul, Porto Alegre (RS). P.137.

Silva, S. L. da; Ferreira, A. de Q.; Pimentei, V. Ma.; Silva, D. K. V. Da; Moura, P. F.; Fernandes, M. L. B. \& Chaves, A. de C. 2004. Identificação enzimática do aparelho digestivo do ouriço-do-mar (Echinometra lucunter) de duas regiões do litoral sul de Pernambuco. Resumos do Congresso Brasileiro de Oceanografia-XVI Semana Nacional de Oceanografia - UNIVALI - Itajaí - SC. P. 222.

Tommasi, L. R. 1965. Alguns Amphiuridae (Ophiuroidea) do litoral de São Paulo e Santa Catarina. Contrições Inst. Oceanogr., Univ. S. Paulo, ser. Ocean. Biol., 8,9 pgs, 5 figs.

Tommasi, L. R. 1999. Echinodermata recentes e fósseis do Brasil. Disponível em: http:// 
www.bdt.fat.org.br/zoologia/echinodermata/ (Acesso em 05 de outubro de 2004).

Vidolin, D.; Santos, I. A. \& Freire, C. A. 2002. Estudo da regulação osmo-iônica da estrela-do-mar Asterina stellifera e do pepino-do-mar Holothuria grisea em poças de maré: Um estudo de campo. Resumos do XXIV Congresso Brasileiro de Zoologia. Itajaí - SC. P. 266.
Xavier, L. A. R. 2005. Inventário dos Echinodermata da Praia da Paciência, Penha - SC. Monografia de Conclusão de Curso em Ciências Biológicas FURB. 56p.

Submetido: Julho/2009 Revisado: Novembro/2009

Aceito: Fevereiro/2010 